\title{
Relações de Equivalência Após Treino com Pareamento Consistente de Estímulos sob Controle Contextual ${ }^{1}$
}

\author{
Grauben José Alves de Assis², \\ Marcelo Quintino Galvão Baptista, \\ Olívia Misae Kato e Keila Regina Alves. \\ Universidade Federal do Pará
}

\begin{abstract}
RESUMO - Alguns estudos têm produzido relações condicionais que se mostraram também equivalentes, através do treino com pareamento consistente de estímulos. O presente estudo buscou verificar se essas relações podem ocorrer também sob controle contextual. Quatro universitários foram submetidos ao treino $A B, A C$ e $A D$, intercalado aos testes de simetria correspondentes $B A, C A$ e $D A$. Em seguida, ao treino $A D$ e ao teste de simetria $D A$ sob controle contextual. Por último, foram testadas as relações de equivalência $B C, C B, B D, D B, C D$ e $D C$, na ausência de estímulos contextuais, e $D B$ e $D C$, com estímulos de contexto. Os participantes alcançaram o critério de acerto em todos os treinos. Três participantes apresentaram as relações simétricas, na ausência de estímulos contextuais, e a relação $D A$, em sua presença. Estes resultados replicam e expandem estudos anteriores, mostrando que mesmo sem conseqüências diferenciais, é possível obter um desempenho consistente sob controle condicional de segunda ordem.
\end{abstract}

Palavras-chave: equivalência de estímulos; treino com pareamento consistente; controle contextual; humanos.

\section{Equivalence Relations After Stimulus Matching Consistent Training Under Contextual Control}

\begin{abstract}
Some studies, using stimulus matching consistent training, had produced conditional relations that have also yielded equivalence relations. The objective of this experiment was to ascertain whether the same type of discriminations would occur under contextual control. Four university students were submitted to sequential relationships. $A B, A C$, and $A D$, interspersed with corresponding tests for symmetry: $B A, C A$, and $D A$. Subsequently, $A D$ was tested with the $D A$ test for symmetry. Finally, the equivalence relations $B C, C B, B D, D B, C D$, and $D C$ were evaluated without the contextual stimuli, whereas the relations $D B$ and $D C$ were tested with contextual stimuli. The participants reached the criterion for accuracy in all training sessions. Three participants presented symmetrical relations without contextual stimuli, while the $D A$ relationship was obtained in the presence of contextual cues. These results replicate and extend previous studies showing that even without differential consequences, it was possible to obtain consistent performance under second-order conditional control.
\end{abstract}

Key Words: stimulus equivalence; matching consistency training; contextual control; humans.

Existem diferenças na literatura quanto às definições de estímulo reforçador e discriminativo. Para Reynolds (1975, p. 9) é o seguinte o entendimento de reforço: "se o aparecimento de um estímulo como conseqüência de uma resposta resulta no aumento da probabilidade de sua ocorrência no futuro, o estímulo é chamado reforçador positivo"3. Definições semelhantes foram propostas por Catania (1968), Skinner (1953) e Sulzer-Azaroff e Mayer (1977). Schlinger Jr., Blakely, Fillhard, e Poling (1991), fizeram

1 Apoio do CNPq. através de bolsa de produtividade ao primeiro autor (processo $n^{\circ}$ 521253/97). Os autores agradecem ao prof. Dr. Olavo Galvão pelas sugestões e a Ane Margareth Monte Verde Silva pelo desenvolvimento do software de controle e análise de dados comportamentais.

2 Endereço: Trav. Castelo Branco, 1923/301, Belém-PA. CEP: 66.063 .420 grauben@amazon.com.br

3 If the appearance of a stimulus as a consequence of a response results in an increased probability that the response will reoccur in the future, the stimulus is called a positive reinforcing stimulus. (Reynolds, G. 1975, p. 9) uma extensa revisão do assunto, propondo a seguinte definição de reforço: "qualquer estímulo apresentado imediatamente após uma resposta, aumenta a freqüência desse desempenho ou da taxa de respostas" (p154). A consideração do termo "imediatamente" parece introduzir uma limitação à definição de estímulo reforçador, sugerindo que somente pode ser considerado como tal aquele estímulo apresentado imediatamente após à resposta. Schoenfeld (1995), também numa interessante revisão a respeito do tema, enfatizou que qualquer estímulo pode ter uma função reforçadora, desde que haja uma identificação de parâmetros, tais como: características especiais do sujeito; intensidade do estímulo; modalidade sensorial e esquemas de aplicação. Porém, ele prossegue dizendo que "o uso do reforço ainda é realmente pragmático pois o experimentador faz sua seleção e aplicação com base em sua experiência. Seu sucesso é julgado após a demonstração pelo sujeito de que "aprendeu" ou tenha "sido condicionado" (p.184)

Quanto à definição de estímulo discriminativo, verifica-se também que há discordâncias. Na literatura, faz-se 
referência à exigência de uma contiguidade temporal entre o estímulo discriminativo $\left(\mathrm{S}^{\mathrm{d}}\right)$ e o comportamento que ele controla para o seu estabelecimento, portanto, exigência de uma maior probabilidade de ocorrência da resposta na presença do que na ausência de um $\mathrm{S}^{\mathrm{d}}$ (Catania, 1984; Reynolds, 1975; Rilling, 1977). Porém, quando se discute especialmente o controle verbal sobre o comportamento, a proximidade temporal entre o estímulo e a resposta nem sempre se evidencia. É o que parece verificar-se no caso do controle sobre o comportamento exercido por regras (enquanto estímulos discriminativos). Em outras palavras: as regras, como estímulos discriminativos (na forma de provérbios, ordens, máximas, leis etc), produzem efeitos que ocorrem indiretamente ou com atraso, observados, muitas vezes, após horas ou vários dias.

Mesmo havendo algumas divergências sobre as definições em questão, discriminações condicionais têm sido ensinadas, tradicionalmente, com o uso de reforço explícito ou consequiências diferenciais em pesquisas sobre equivalência de estímulos. Porém, algumas pesquisas sobre equivalência, têm sido conduzidas mais recentemente utilizando um procedimento de pareamento consistente entre os estímulos modelo e de comparação correto, na ausência de consequiências diferenciais imediatas (Álvares, 1996; Assis, Baptista, Damin \& Álvares, 1997; Baptista \& Assis, 1995; Barros, Baptista \& Assis, 1998; Damin, Assis \& Baptista, 1998; Harrison \& Green, 1990).

O que caracteriza o treino de pareamento consistente entre estímulos (conforme definido no estudo de Baptista \& Assis, 1995), é o fato do estímulo de comparação correto ser apresentado em todas as tentativas de um bloco, juntamente com o modelo e pelo menos dois outros estímulos incorretos $v a$ riando ao longo do treino. Em cada tentativa, a resposta a quaisquer dos estímulos, correto ou incorreto, produz sempre uma nova configuração dos mesmos sem conseqüências diferenciais imediatas. Portanto, para cada modelo, o estímulo de comparação correto é sempre apresentado e os dois incorretos mudam a cada duas tentativas consecutivas, sendo um destes substituído por outro. Neste tipo de treino sem conseqüências diferenciais para respostas corretas e incorretas a cada tentativa, o pareamento consistente modelo-estímulo de comparação correto parece tornar mais provável a escolha do estímulo correto, em detrimento da escolha de quaisquer estímulos incorretos não pareados de forma consistente com o modelo. Na literatura, este tipo de estrutura de treino das relações condicionais é relatado por Saunders, Saunders, Kirby \& Spradlin (1988) e Harrison \& Green (1990, Experimento 2). Outros estudos replicaram posteriormente aqueles, refinando o procedimento de treino com pareamento consistente (Álvares, 1996; Assis \& cols., 1997; Baptista \& Assis, 1995; Barros \& cols., 1998; Damin \& cols., 1998).

4 The actual uses of "reinforcement" are still in the pragmatic realm where an experimenter makes his selections and applications on the basis of common experience. His success is judged after the fact by the demonstration that his subject has "learned" or "been conditioned." (Schoenfeld, W. 1995, p. 184).
Em todos os estudos conduzidos com pareamento consistente entre estímulos, sem consequiências diferenciais imediatas, foi usada uma contingência de quatro termos. $\mathrm{O}$ presente estudo caracteriza-se pela introdução de um quinto elemento à contingência (contingência de cinco termos) e é, portanto, uma ampliação da unidade básica de análise do comportamento, conforme foi proposta por Sidman (1986). Nessa contingência assim ampliada, o quinto elemento assume um controle condicional denominado de segunda ordem, ou controle contextual, sobre a discriminação condicional (ver Assis \& Galvão, 1996; Lazar \& Kotlarchyk, 1986; Bush, Sidman \& de Rose, 1989; Gatch \& Osborne, 1989; Lynch \& Green, 1991; Wulfert \& Hayes, 1988). Estes estudos investigaram o problema de se e como os estímulos contextuais, isoladamente, controlam grupos de discriminações condicionais, que envolvem relações de equivalência. A ampliação da unidade básica do comportamento através do uso do controle contextual proporciona instrumentos que permitem analisar a amplitude e a complexidade da variação comportamental.

Os estudos conduzidos sem conseqüências diferenciais imediatas indicaram ser o pareamento consistente entre estímulos uma variável alternativa de controle do comportamento. $\mathrm{O}$ objetivo do presente estudo foi verificar o estabelecimento de discriminações condicionais sob controle contextual através de um treino por pareamento consistente entre o modelo e o estímulo de comparação correto, na ausência de consequiências diferenciais imediatas para respostas corretas e incorretas. O presente estudo visou, ainda, investigar se estas relações condicionais constituíam relações simétricas e de equivalência, e se permaneciam sob controle contextual.

\section{Método}

\section{Participantes}

Quatro universitários, de diferentes cursos de graduação (exceto Psicologia), de ambos os sexos, com idade variando de 17 a 21 anos, foram recrutados para o experimento, através de contatos pessoais. Após cada sessão, os participantes recebiam um lanche. No final do experimento, todos receberam $\mathrm{R} \$ 10,00$ (dez reais) pela participação.

\section{Situação experimental e equipamento}

As sessões foram realizadas em uma sala do Laboratório de Psicologia Experimental da Universidade Federal do Pará de aproximadamente $6 \mathrm{~m}^{2}$. O experimentador permanecia em uma sala adjacente, separada por um vidro que permitia uma visão unilateral, de onde monitorava o desempenho dos participantes.

As sessões foram conduzidas em um microcomputador (modelo IBM PENTIUM de $130 \mathrm{MHz}$ ) que controlava e registrava as respostas corretas e incorretas, apresentação dos estímulos (modelo e de comparação), número de tentativas, através de um programa escrito em linguagem VISU- 


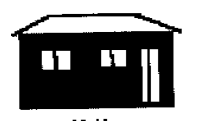

(A1)

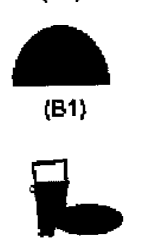

[C1)

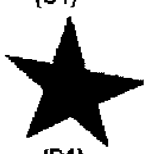

(D1)

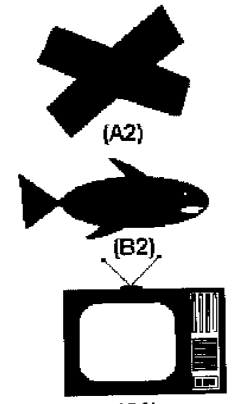

(C2)

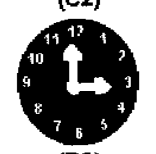

(D2)

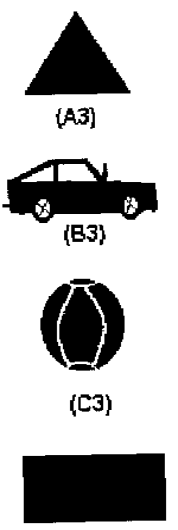

(D3)

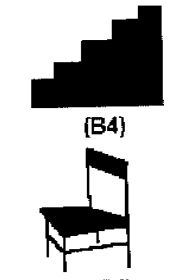

(C4)

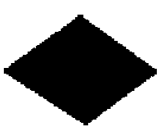

(D4)
Figura 1. Conjunto de estímulos usados no experimento

AL BASIC 5.0, desenvolvido para essa finalidade. Ao microcomputador estava conectado um monitor SVGA de 14" com tela sensível ao toque (VGART, policromático, modelo 1448T).

\section{Estímulos}

No pré-treino, foram usados estímulos não experimentais. No treino e nos testes, foram usadas formas geométricas usuais e figuras de objetos do cotidiano (ver Figura 1), além das cores verde e vermelho como estímulos contextuais (ver Figura 2).

\section{Procedimento}

Foram programadas três fases. O Quadro 1 apresenta a seqüência de treino e testes nas Fases 1, 2 e 3, especificando as relações ensinadas e testadas correspondentes.

$\mathrm{Na}$ Fase 1, foi conduzido o treino das relações condicionais $\mathrm{AB}, \mathrm{AC}$ e $\mathrm{AD}$ intercalado com o teste das respectivas relações simétricas.

$\mathrm{Na}$ Fase 2, a discriminação condicional AD foi colocada sob controle contextual e o controle contextual da relação simétrica DA foi testado.

$\mathrm{Na}$ Fase 3, foram conduzidos os testes de equivalência $\mathrm{BC}, \mathrm{CB}, \mathrm{CD}$ e $\mathrm{DC}$, precedidos pelos treinos mistos das respectivas discriminações condicionais ( $\mathrm{AB}, \mathrm{AC}$ e $\mathrm{AD})$, necessárias para a emergência dessas relações de equivalência testadas. Foram aplicados, ainda, os testes do controle contextual das relações de equivalência DB e DC, após o estabelecimento do controle contextual das discriminações condicionais AD.

O Quadro 2 apresenta a seqüência de treino e testes da Fase 1, com o número de tentativas por tipo de tentativas em cada bloco. Foram programados nove tipos de tentativas, sendo três tipos para cada modelo (A1, A2 e A3). Nos treinos mistos, os tipos de tentativas para cada discriminação condicional ensinada (por exemplo, A1B1, A2B2 e A3B3) foram misturados e o número de tentativas para cada tipo foi reduzido.
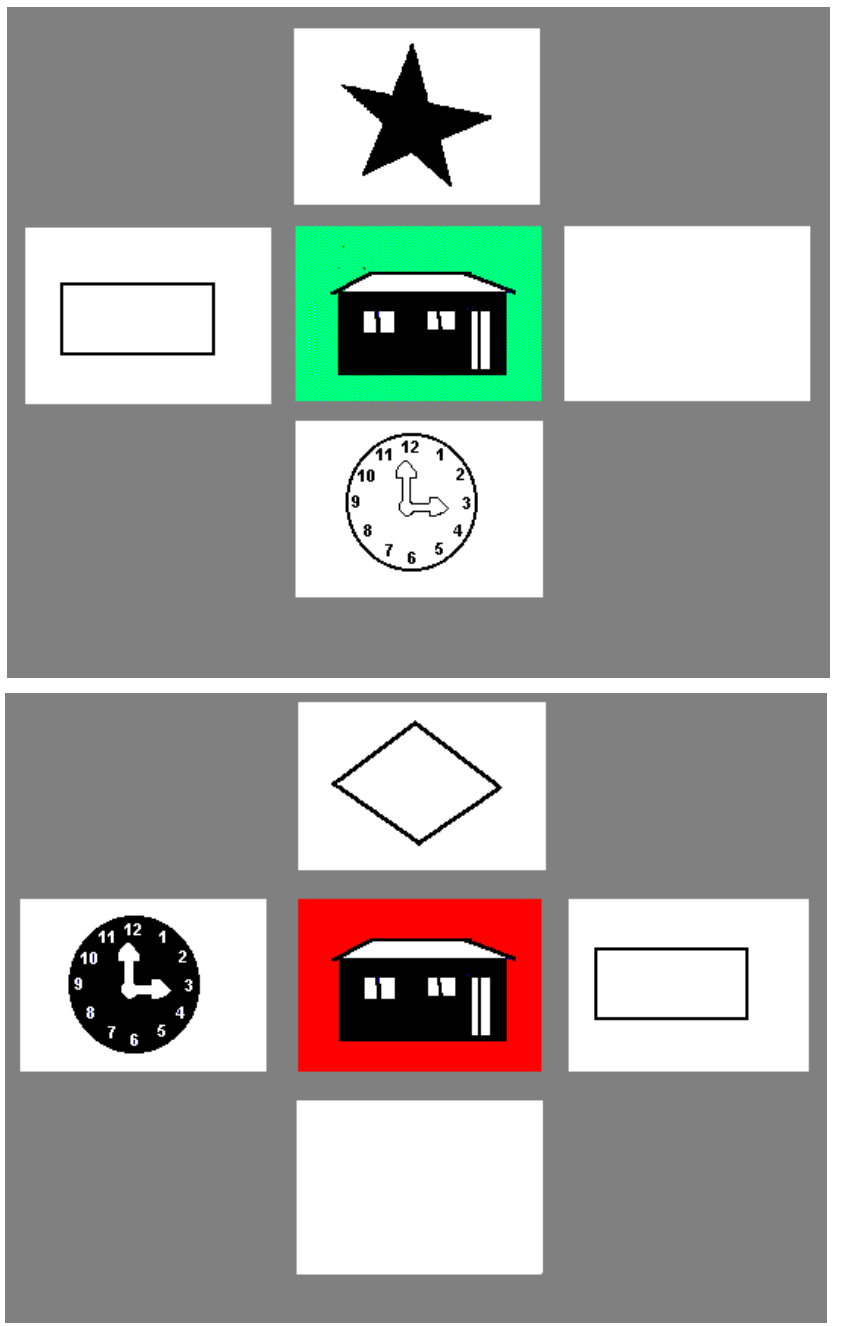

Figura 2. Exemplo de duas tentativas da relação AD com estímulo contextual (desenho de uma casa com o fundo verde ou vermelho na janela central), e estímulo de comparação correto (desenhos de uma estrela e um relógio preenchidos).

O treino iniciava com a apresentação do modelo na " janela" central onde permanecia até que o participante a tocasse, produzindo o desaparecimento do modelo, por 1 segundo; após isto, o modelo era reapresentado, na mesma janela, juntamente com três estímulos de comparação em três das quatro "janelas" laterais. Os estímulos desapareciam, após a resposta de escolha a um dos três estímulos de comparação, por 1 segundo (intervalo entre tentativas), sendo o mesmo modelo reapresentado. Depois de uma nova resposta ao modelo, outra configuração de apresentação dos estímulos de comparação aparecia, e assim por diante, até que fosse completado um bloco de 36 tentativas. Uma “janela" ficava desativada (permanecendo apenas o seu contorno na tela). Qualquer resposta ao modelo, após a apresentação dos estímulos de comparação, ou à "janela" desativada não alterava a configuração da tela.

Em todas as tentativas, havia um estímulo de comparação correto apresentado de forma consistente com o modelo, acompanhados de dois outros estímulos de comparação incorretos, não pareados consistentemente com o modelo. 
G. J. A. Assis \& cols.

Quadro 1. Sequiência de treino e teste das relações condicionais ensinadas e testadas sem ou com controle contextual das cores verde (vd) e vermelha (vm).

\begin{tabular}{|c|c|c|}
\hline \multirow[t]{4}{*}{ Fase 1: } & \multicolumn{2}{|l|}{ sem controle contextual } \\
\hline & Treino A B - Simetria BA & A 1B 1-B 1A 1, A 2B 2-B 2A 2, А 3В 3-B 3А 3 \\
\hline & Treino AC - Simetria CA & A $1 C 1-C 1 A 1, A 2 C 2-C 2 A 2, A 3 C 3-C 3 A 3$ \\
\hline & Treino AD - Simetria DA & A 1D 1-D 1A 1, A 2D 2-D 2A 2, A 3D 3-D 3A 3 \\
\hline \multirow[t]{7}{*}{ Fase 2: } & \multicolumn{2}{|l|}{ controle contextual (vd/vm) } \\
\hline & \multirow{4}{*}{ Treino AD - Simetria DA } & A 1D 1-D 1A 1(vd), A 1D 2-D 2A 1(vm) \\
\hline & & A 2D 2-D 2A 2(vd), A 2D 1-D 1A 2(vm) \\
\hline & & A 2D 3-D 3A 2(vm), A 3D 3-D 3A 3(vd) \\
\hline & & A 3D 2-D 2A 3(vm) \\
\hline & Treino M isto A D & A 1D 1-A 2D 2-A 3D 3(vd), A 1D 2-A 2D 1-A 2D 3-A 3D 2(vm) \\
\hline & Simetria DA & D 1A 1-D 2A 2-D 3A 3(vd), D 2A 1-D 1A 2-D 3A 2-D 2A 3(vm) \\
\hline \multirow[t]{14}{*}{ Fase 3: } & \multicolumn{2}{|l|}{ a) sem controle contextual } \\
\hline & Treino $M$ isto $A B$ e AC & A 1B 1-A 2B 2-A 3B 3, A 1C 1-A 2C 2-A 3С 3 \\
\hline & Equivalência BC-CB & В 1C1-B 2C2-B 3C 3, C1B 1-С 2B 2-С 3B 3 \\
\hline & Treino $M$ isto $A B$ e $A D$ & A 1B 1-A 2B 2-A 3B 3, A 1D 1-A 2D 2-A 3D 3 \\
\hline & Equivalência BD-DB & B 1D 1-B 2D 2-B 3D 3, D 1B 1-D 2B 2-D 3B 3 \\
\hline & \multicolumn{2}{|c|}{ b) com controle contextual (vd/vm) } \\
\hline & Treino M isto AD & A 1D 1-A 2D 2-A 3D 3(vd), A 1D 2-A 2D 1-A 2D 3-A 3D 2(vm) \\
\hline & Equivalência DB & D 1B 1-D 2B 2-D 3B 3(vd) \\
\hline & \multicolumn{2}{|l|}{ c) sem controle contextual } \\
\hline & Treino M isto AC e AD & A 1C 1-A 2C2-A 3C 3, A 1D 1-A 2D 2-A 3D 3 \\
\hline & Equivalência CD-DC & C1D1-C2D2-C3D 3, D 1C1-D 2C2-D 3C3 \\
\hline & \multicolumn{2}{|c|}{ d) com controle contextual (vd/vm) } \\
\hline & Treino M isto AD & A 1D 1-A 2D 2-A 3D 3(vd), A 1D 2-A 2D 1-A 2D 3-A 3D 2 (vm) \\
\hline & Equivalência DC & D1C1-D2C2-D3C3 (vm) \\
\hline
\end{tabular}

A escolha do estímulo de comparação que era pareado sistematicamente com o modelo era registrada como resposta correta; a escolha de quaisquer estímulos de comparação inconsistentes era registrada como resposta incorreta. Não foram programadas conseqüências diferenciais imediatas para as respostas corretas e incorretas. Para efeito de balanceamento dos tipos de tentativas, foi usado, durante o treino, um estímulo de distração, que substituiu um dos estímulos de comparação incorreto em cada discriminação condicional. Na primeira tentativa (por exemplo, do tipo A1... B1 B2 B3), A1 era o modelo, B1 o estímulo de comparação correto pareado consistentemente com o modelo, e B2 e B3 os estímulos de comparação incorretos. Um dos estímulos de comparação incorretos (B2, por exemplo) permanecia em duas tentativas consecutivas e ficava ausente na próxima, quando era então substituído por um outro estímulo de comparação (B4). O outro estímulo de comparação incorreto (B3, por exemplo) era apresentado junto do primeiro na Tentativa 1 , substituído por outro estímulo na Tentativa 2, e era reapresentado consecutivamente nas Tentativas 3 e 4 , e assim por diante.

Todos os blocos de treino e de testes eram compostos de 36 tentativas. O critério de mudança de um bloco de treino para outro e para os testes, consistia de um erro, no máximo, em cada bloco ( 35 acertos sobre 36 tentativas), correspondendo a $97 \%$ de acertos. Foram preparadas sequiências randomizadas de 72 tentativas, sendo que as 36 tentativas iniciais formavam um bloco e, em caso de repetição do bloco, eram usadas as 36 tentativas seguintes (da $37^{\mathrm{a}}$ à $72^{\mathrm{a}}$ tentativa). Se não atingisse o critério de treino em um bloco, o participante era reexposto ao mesmo até 10 vezes, o que caracterizava a persistência no erro, sendo, portanto, apresentado o bloco de treino seguinte, após essa máxima reexposição.

Os testes de simetria foram aplicados de forma intercalada aos treinos (por exemplo, a simetria B1A1 era aplicada após o treino da relação A1B1 e antes da A2B2, e assim por diante, conforme mostra o Quadro 2).

$\mathrm{Na}$ Fase 2, foi realizado o treino AD, aplicado o teste de simetria DA e o treino misto AD e simetria DA com estímulos contextuais (ver Quadro 3). Nesse treino, foram usados como estímulos contextuais as cores verde e vermelha. Por exemplo, nas tentativas do tipo A1 D1 D2D3, o modelo A1 sempre era apresentado na janela central, com fundo verde, enquanto nas tentativas do tipo Al D2 D1D4, o modelo A1 sempre era apresentado também na janela central, mas com fundo vermelho (ver Figura 2).

Na Fase 3, foram aplicados os testes de equivalência $\mathrm{BC}, \mathrm{CB}, \mathrm{BD}, \mathrm{DB}, \mathrm{CD}$ e $\mathrm{DC}$, intercalados aos treinos mistos correspondentes (ver Quadro 4). Também ocorreu o treino misto AD com estímulos contextuais, seguido dos testes de equivalência DB e DC com estímulos contextuais. As sessões experimentais tinham uma duração máxima de 30 minutos. No final de cada sessão, uma mensagem aparecia na tela: "Parabéns, você chegou ao final das atividades. Chame o experimentador. Muito obrigado!".

Após o término do experimento, perguntava-se ao participante: "O que você estava fazendo?" 
Quadro 2. Treino das relações condicionais $A B, A C$ e $A D$, testes de simetria $B A, C A$ e $D A$ sem estímulos contextuais, com o tipo e número de tentativas.

\begin{tabular}{|c|c|c|c|c|c|c|}
\hline \multirow{2}{*}{ R elação } & \multirow{2}{*}{$\begin{array}{l}\text { E stímulos } \\
\text { C ontextuais }\end{array}$} & \multirow{2}{*}{\multicolumn{3}{|c|}{ Tipo de Tentativas }} & \multicolumn{2}{|c|}{$\mathrm{N} \cong$ de Tentativas } \\
\hline & & & & & \multirow{2}{*}{$\begin{array}{c}\begin{array}{c}\text { Total } \\
\text { Parcial }\end{array} \\
12\end{array}$} & $\begin{array}{l}\text { Total } \\
\text { Geral }\end{array}$ \\
\hline \multirow[t]{3}{*}{$\overline{A B}: A 1 B 1$} & & A 1 & B1+ & B 2B 3 & & \\
\hline & & & $\mathrm{B} 1+$ & B 2B 4 & 12 & \\
\hline & & & B $1+$ & B 3B 4 & 12 & 36 \\
\hline SIMET B $1 A 1$ & & B 1 & A $1+$ & A 2A 3 & 36 & 36 \\
\hline \multirow[t]{3}{*}{$\mathrm{AB}: \mathrm{A} 2 \mathrm{~B} 2$} & & A2 & B2+ & B 1B 3 & 12 & \\
\hline & & & B $2+$ & B 1B 4 & 12 & \\
\hline & & & B $2+$ & B 3B 4 & 12 & 36 \\
\hline SIMET B $2 A 2$ & & B2 & A $2+$ & A 1 A 3 & 36 & 36 \\
\hline \multirow[t]{3}{*}{$A B: A 3 B 3$} & & A3 & B 3+ & B 1B2 & 12 & \\
\hline & & & B 3+ & B 1B 4 & 12 & \\
\hline & & & B 3+ & B 2B 4 & 12 & 36 \\
\hline SIMET B $3 A 3$ & & B3 & A3+ & A $1 A 2$ & 36 & 36 \\
\hline$\overline{\mathrm{AB}: \mathrm{MISTA}}$ & & A 1 & B 1t & B2B 4 & 04 & \\
\hline & & & B $1+$ & B 2B 3 & 04 & \\
\hline & & & B $1+$ & B 3B 4 & 04 & \\
\hline & & A 2 & B $2+$ & B 3B 4 & 04 & \\
\hline & & & B $2+$ & B 1B 4 & 04 & \\
\hline & & & B $2+$ & B 1B 3 & 04 & \\
\hline & & A 3 & B 3+ & B 1B 2 & 04 & \\
\hline & & & B 3+ & B 1B 4 & 04 & \\
\hline & & & B $3+$ & B 2B 4 & 04 & 36 \\
\hline SIMET BA MISTA & & B 1 & A $1+$ & A 2 A 3 & 12 & \\
\hline & & B2 & $A 2+$ & A $1 A 3$ & 12 & \\
\hline & & B3 & A $3+$ & A $1 A 2$ & 12 & 36 \\
\hline$\overline{A C}: A 1 C 1$ & & A 1 & $\mathrm{C} 1+$ & $\mathrm{C} 2 \mathrm{C} 3$ & 12 & \\
\hline & & & $\mathrm{C} 1+$ & $\mathrm{C} 2 \mathrm{C} 4$ & 12 & \\
\hline & & & $\mathrm{C} 1+$ & $\mathrm{C} 3 \mathrm{C} 4$ & 12 & 36 \\
\hline SIMET CIA 1 & & C1 & A $1+$ & $\mathrm{A} 2 \mathrm{~A} 3$ & 36 & 36 \\
\hline $\mathrm{AC}: \mathrm{A} 2 \mathrm{C} 2$ & & A2 & $\mathrm{C} 2+$ & C1C3 & 12 & \\
\hline & & & $\mathrm{C} 2+$ & $\mathrm{C} 1 \mathrm{C} 4$ & 12 & \\
\hline & & & $\mathrm{C} 2+$ & $\mathrm{C} 3 \mathrm{C} 4$ & 12 & 36 \\
\hline SIMET C $2 A 2$ & & $\mathrm{C} 2$ & A 2+ & A 1A 3 & 36 & 36 \\
\hline $\mathrm{AC}: \mathrm{A} 3 \mathrm{C} 3$ & & A3 & C3+ & C1C2 & 12 & \\
\hline & & & $\mathrm{C} 3+$ & $\mathrm{C} 1 \mathrm{C} 4$ & 12 & \\
\hline & & & $\mathrm{C} 3+$ & $\mathrm{C} 2 \mathrm{C} 4$ & 12 & 36 \\
\hline SIMET C 3 A 3 & & C3 & A3+ & A $1 A 2$ & 36 & 36 \\
\hline$\overline{\mathrm{AC}: \mathrm{MISTA}}$ & & A 1 & $\mathrm{C} 1+$ & $\mathrm{C} 2 \mathrm{C} 4$ & 04 & \\
\hline & & & $\mathrm{C} 1+$ & $\mathrm{C} 2 \mathrm{C} 3$ & 04 & \\
\hline & & & $\mathrm{C} 1+$ & $\mathrm{C} 3 \mathrm{C} 4$ & 04 & \\
\hline & & A 2 & $\mathrm{C} 2+$ & $\mathrm{C} 3 \mathrm{C} 4$ & 04 & \\
\hline & & & $\mathrm{C} 2+$ & $\mathrm{C} 1 \mathrm{C} 4$ & 04 & \\
\hline & & & $\mathrm{C} 2+$ & $\mathrm{C} 1 \mathrm{C} 3$ & 04 & \\
\hline & & A 3 & $\mathrm{C} 3+$ & $\mathrm{C} 1 \mathrm{C} 2$ & 04 & \\
\hline & & & $\mathrm{C} 3+$ & $\mathrm{C} 1 \mathrm{C} 4$ & 04 & \\
\hline & & & $\mathrm{C} 3+$ & $\mathrm{C} 2 \mathrm{C} 4$ & 04 & 36 \\
\hline SIMET CA MISTA & & C1 & A $1+$ & A2A3 & 12 & \\
\hline & & $\mathrm{C} 2$ & A $2+$ & A $1 A 3$ & 12 & \\
\hline & & $\mathrm{C} 3$ & A 3+ & A $1 A 2$ & 12 & 36 \\
\hline$\overline{A D}:$ A 1D 1 & & A 1 & D 1+ & D2D 3 & 12 & \\
\hline & & & D $1+$ & $\mathrm{D} 2 \mathrm{D} 4$ & 12 & \\
\hline & & & D $1+$ & D 3D 4 & 12 & 36 \\
\hline SIMET D $1 A 1$ & & D1 & A $1+$ & A2A3 & 36 & 36 \\
\hline AD: A 2D2 & & A2 & $\mathrm{D} 2+$ & D1D3 & 12 & \\
\hline & & & $\mathrm{D} 2+$ & D1D 4 & 12 & \\
\hline & & & $\mathrm{D} 2+$ & D 3D 4 & 12 & 36 \\
\hline SIMET D $2 A 2$ & & D2 & A 2+ & A 1 A 3 & 36 & 36 \\
\hline$\overline{A D: A 3 D 3}$ & & A3 & D3+ & D1D2 & 12 & \\
\hline & & & D3+ & D1D 4 & 12 & \\
\hline & & & D3+ & D2D 4 & 12 & 36 \\
\hline SIMET D $3 A 3$ & & D3 & A3+ & A 1A 2 & 36 & 36 \\
\hline$\overline{A D ~ M I S T A}$ & & A 1 & D 1+ & D2D 4 & 04 & \\
\hline & & & DIt & $\mathrm{D} 2 \mathrm{D} 3$ & 04 & \\
\hline & & & D $1+$ & D 3D 4 & 04 & \\
\hline & & A 2 & $\mathrm{D} 2+$ & D 3D 4 & 04 & \\
\hline & & & D2+ & D1D 4 & 04 & \\
\hline & & & $\mathrm{D} 2+$ & D1D 3 & 04 & \\
\hline & & A 3 & D3+ & D1D 2 & 04 & \\
\hline & & & D3+ & D 1D 4 & 04 & \\
\hline & & & D3+ & D2D 4 & 04 & 36 \\
\hline SIMET DA MISTA & & D1 & A $1+$ & A 2 A 3 & 12 & \\
\hline & & $\mathrm{D} 2$ & A $2+$ & A 1 A 3 & 12 & \\
\hline & & D3 & A $3+$ & $A 1 A 2$ & 12 & 36 \\
\hline
\end{tabular}

Quadro 3. Treino de discriminações condicionais $A D$ e simetria $D A$, Treino Misto $A D$ e simetria $D A$ com estímulos contextuais.

\begin{tabular}{|c|c|c|c|c|c|}
\hline \multirow{2}{*}{ R elação } & \multirow{2}{*}{$\begin{array}{l}\text { E stímulos } \\
\text { C ontextuais }\end{array}$} & \multirow{2}{*}{\multicolumn{2}{|c|}{ Tipo de Tentativas }} & \multicolumn{2}{|c|}{ № de Tentativas } \\
\hline & & & & $\begin{array}{l}\text { Total } \\
\text { Parcial }\end{array}$ & $\begin{array}{l}\text { Total } \\
\text { Geral }\end{array}$ \\
\hline \multirow[t]{3}{*}{$\overline{A D}: A 1 D 1$} & Verde & A 1 & $\mathrm{D} 1+\mathrm{D} 2 \mathrm{D} 3$ & 12 & \\
\hline & & A 1 & $\mathrm{D} 1+\mathrm{D} 2 \mathrm{D} 4$ & 12 & \\
\hline & & A 1 & $D 1+D 3 D 4$ & 12 & 36 \\
\hline SIMET DIA 1 & Verde & D1 & $A 1+A 2 A 3$ & 36 & 36 \\
\hline \multirow{3}{*}{$\overline{A D}:$ A 1D2 } & Vermelho & A1 & $\mathrm{D} 2+\mathrm{D} 1 \mathrm{D} 3$ & 12 & \\
\hline & & A 1 & $\mathrm{D} 2+\mathrm{D} 1 \mathrm{D} 4$ & 12 & \\
\hline & & A 1 & $D 2+D 3 D 4$ & 12 & 36 \\
\hline SIMET D 2A 1 & Vermelho & D2 & $A 1+A 2 A 3$ & 36 & 36 \\
\hline \multirow[t]{3}{*}{$\overline{A D}: A 2 D 1$} & Vermelho & A2 & $\mathrm{D} 1+\mathrm{D} 2 \mathrm{D} 3$ & 12 & \\
\hline & & A2 2 & $\mathrm{D} 1+\mathrm{D} 2 \mathrm{D} 4$ & 12 & \\
\hline & & A 2 & $D 1+D 3 D 4$ & 12 & 36 \\
\hline SIMET D $1 A 2$ & Vermelho & D1 & $A 2+A 1 A 3$ & 36 & 36 \\
\hline \multirow[t]{3}{*}{$\overline{A D}: A 2 D 2$} & Verde & A2 & D2+ D1D3 & 12 & \\
\hline & & A 2 & $\mathrm{D} 2+\mathrm{D} 1 \mathrm{D} 4$ & 12 & \\
\hline & & A 2 & $D 2+D 3 D 4$ & 12 & 36 \\
\hline SIMET D 2A 2 & Verde & D2 & $A 2+A 1 A 3$ & 36 & 36 \\
\hline \multirow[t]{3}{*}{ AD: A 2D 3} & Vermelho & A2 & D3+ D1D2 & 12 & \\
\hline & & A2 & D3+ D1D4 & 12 & \\
\hline & & A 2 & $\mathrm{D} 3+\mathrm{D} 2 \mathrm{D} 4$ & 12 & 36 \\
\hline SIMET D $3 A 2$ & Vermelho & D3 & $A 2+A 1 A 3$ & 36 & 36 \\
\hline \multirow[t]{3}{*}{$\overline{A D}:$ A 3D 2} & Vermelho & A3 & $\mathrm{D} 2+\mathrm{D} 1 \mathrm{D} 3$ & 12 & \\
\hline & & & $\mathrm{D} 2+\mathrm{D} 1 \mathrm{D} 4$ & 12 & \\
\hline & & & $\mathrm{D} 2+\mathrm{D} 3 \mathrm{D} 4$ & 12 & 36 \\
\hline SIMET D 2A 3 & Vermelho & D2 & $A 3+A 1 A 2$ & 36 & 36 \\
\hline \multirow{3}{*}{ AD: A 3D 3} & Verde & A3 & D3+ D1D2 & 12 & \\
\hline & & A 3 & D3+ D1D4 & 12 & \\
\hline & & A 3 & D $3+D 3 D 4$ & 12 & 36 \\
\hline SIMET D $3 A 3$ & Verde & D3 & $A 3+A 1 A 2$ & 36 & 36 \\
\hline$\overline{A D}$ MISTA & & & & & \\
\hline \multirow[t]{3}{*}{ A $1 D 1$} & Verde & A 1 & $\mathrm{D} 1+\mathrm{D} 2 \mathrm{D} 3$ & 02 & \\
\hline & & & $\mathrm{D} 1+\mathrm{D} 2 \mathrm{D} 4$ & 02 & \\
\hline & & & $\mathrm{D} 1+\mathrm{D} 3 \mathrm{D} 4$ & 02 & \\
\hline \multirow[t]{3}{*}{$A 1 D 2$} & Vermelho & A 1 & $\mathrm{D} 2+\mathrm{D} 1 \mathrm{D} 3$ & 02 & \\
\hline & & & $\mathrm{D} 2+\mathrm{D} 1 \mathrm{D} 4$ & 02 & \\
\hline & & & $D 2+D 3 D 4$ & 02 & \\
\hline \multirow[t]{3}{*}{ A 2D 1} & Vermelho & A2 & $\mathrm{D} 1+\mathrm{D} 2 \mathrm{D} 3$ & 02 & \\
\hline & & & $\mathrm{D} 1+\mathrm{D} 2 \mathrm{D} 4$ & 02 & \\
\hline & & & $D 1+D 3 D 4$ & 02 & \\
\hline \multirow[t]{3}{*}{ A 2D 2} & Verde & A2 & D2+ D1D3 & 02 & \\
\hline & & & $\mathrm{D} 2+\mathrm{D} 1 \mathrm{D} 4$ & 02 & \\
\hline & & & $D 2+D 3 D 4$ & 02 & \\
\hline \multirow[t]{3}{*}{ A 2D 3} & Vermelho & A2 & D3+ D1D2 & 02 & \\
\hline & & & D3+ D1D4 & 02 & \\
\hline & & & $\mathrm{D} 3+\mathrm{D} 2 \mathrm{D} 4$ & 02 & \\
\hline \multirow[t]{3}{*}{ A 3D 2} & Vermelho & A3 & D2+ D1D3 & 02 & \\
\hline & & & $\mathrm{D} 2+\mathrm{D} 1 \mathrm{D} 4$ & 02 & \\
\hline & & & $\mathrm{D} 2+\mathrm{D} 3 \mathrm{D} 4$ & 02 & 36 \\
\hline \multicolumn{6}{|l|}{ SIMET DA } \\
\hline D $1 A 1$ & Verde & D1 & $\mathrm{A} 1+\mathrm{A} 2 \mathrm{~A} 3$ & 36 & 36 \\
\hline D1A 2 & Vermelho & D1 & $A 2+A 1 A 3$ & 36 & 36 \\
\hline D2A 1 & Vermelho & D2 & $A 1+A 2 A 3$ & 36 & 36 \\
\hline D2A 2 & Verde & D2 & $A 2+A 1 A 3$ & 36 & 36 \\
\hline D2A 3 & Vermelho & D2 & $A 3+A 1 A 2$ & 36 & 36 \\
\hline D 3 A 2 & Vermelho & D3 & $A 2+A 1 A 3$ & 36 & 36 \\
\hline
\end{tabular}

\section{Resultados}

A Tabela 1 apresenta o desempenho dos quatro participantes nos blocos de treino misto e nos blocos de testes de simetria e de equivalência, com e sem controle contextual. Todos alcançaram o critério de acerto no treino das discriminações condicionais e foram expostos aos testes correspondentes, após cada discriminação condicional ensinada.

Os relatos dos participantes em entrevistas pós-experimentais foram consistentes com o desempenho no treino 
Quadro 4. - Treino Misto $A B, A C$ e $A D$ com os testes de equivalência correspondentes e Treino Misto $A D$ com estímulos contextuais e os testes de equivalência $D B$ e $D C$ com estímulos contextuais.

\begin{tabular}{|c|c|c|c|c|c|c|}
\hline \multirow{4}{*}{$\begin{array}{l}\text { Re elação } \\
\mathrm{AB} \text { e AC MISTAS }\end{array}$} & \multirow{2}{*}{$\begin{array}{l}\text { E stímulos } \\
\text { Contextuais }\end{array}$} & \multirow{2}{*}{\multicolumn{3}{|c|}{ Tipo de Tentativas }} & \multicolumn{2}{|c|}{ № de Tentativas } \\
\hline & & & & & $\begin{array}{c}\text { Total } \\
\text { Parcial }\end{array}$ & \multirow[t]{2}{*}{$\begin{array}{l}\text { Total } \\
\text { Geral } \\
\end{array}$} \\
\hline & & \multicolumn{4}{|c|}{ (Ver Fase2) } & \\
\hline & & B 1 & $\mathrm{C} 1+$ & $\mathrm{C} 2 \mathrm{C} 3$ & 12 & \\
\hline & & B2 & $\mathrm{C} 2+$ & $\mathrm{C} 1 \mathrm{C} 3$ & 12 & \\
\hline & & B3 & $\mathrm{C} 3+$ & $\mathrm{C} 1 \mathrm{C} 2$ & 12 & 36 \\
\hline EQUIV CB & & $\mathrm{C} 1$ & B1+ & B2B 3 & 12 & \\
\hline & & C2 & B2+ & B 1B 3 & 12 & \\
\hline & & C3 & B $3+$ & B 1B 2 & 12 & 36 \\
\hline$\overline{A B}$ e AD MISTAS & & \multicolumn{4}{|c|}{ (Ver Fase2) } & 36 \\
\hline \multirow[t]{3}{*}{ EQUIV BD } & & B1 & D1+ & D2D 3 & 12 & \\
\hline & & B2 & D2t & D1D 3 & 12 & \\
\hline & & B3 & D3+ & D1D2 & 12 & 36 \\
\hline \multirow[t]{3}{*}{ EQUIV DB } & & D1 & B1+ & B 2B 3 & 12 & \\
\hline & & D2 & B2+ & B 1B 3 & 12 & \\
\hline & & D3 & B $3+$ & B 1B2 & 12 & 36 \\
\hline \multicolumn{7}{|l|}{$\overline{A D}$ MISTA } \\
\hline \multirow[t]{3}{*}{ A 1D 1} & Verde & A 1 & D1+ & D2D 3 & 02 & \\
\hline & & & D1t & D2D 4 & 02 & \\
\hline & & & D1+ & D3D 4 & 02 & \\
\hline A 1D2 2 & Vermelho & A 1 & D2t & D1D3 & 02 & \\
\hline & & & D2+ & D1D 4 & 02 & \\
\hline & & & D2+ & D 3D 4 & 02 & \\
\hline A 2D 1 & Vermelho & $\mathrm{A} 2$ & D1t & D2D3 & 02 & \\
\hline & & & D1+ & D2D 4 & 02 & \\
\hline & & & D1+ & D3D 4 & 02 & \\
\hline A 2D 2 & Verde & A2 & D2t & D1D3 & 02 & \\
\hline & & & D2+ & D1D 4 & 02 & \\
\hline & & & D2+ & D 3D 4 & 02 & \\
\hline A 2D 3 & Vermelho & $\mathrm{A} 2$ & D3t & D1D2 & 02 & \\
\hline & & & D3+ & D1D 4 & 02 & \\
\hline & & & D3+ & D2D 4 & 02 & \\
\hline$A D: A 3 D 2$ & Vermelho & A3 & $\mathrm{D} 2+$ & D1D3 & 02 & \\
\hline & & & D2t & D1D 4 & 02 & \\
\hline & & & D2t+ & D3D 4 & 02 & 36 \\
\hline EQUIV DB & & & & & & \\
\hline D 1B 1 & Verde & D1 & B 1+ & B 2B 3 & 36 & 36 \\
\hline D2B 2 & Verde & D2 & B2+ & B 1B3 & 36 & 36 \\
\hline D 3B 3 & Verde & D3 & B $3+$ & B 1B2 & 36 & 36 \\
\hline$\overline{A C}$ EAD MISTAS & & & & (Ver Fas & & 36 \\
\hline EQUIV CD & & $\mathrm{C} 1$ & D1t & D2D 3 & 12 & \\
\hline & & C2 & D2+ & D1D 3 & 12 & \\
\hline & & $\mathrm{C} 3$ & D3+ & D 1D2 & 12 & 36 \\
\hline EQUIV DC & & D1 & $\mathrm{C} 1+$ & $\mathrm{C} 2 \mathrm{C} 3$ & 12 & \\
\hline & & D2 & $\mathrm{C} 2+$ & $\mathrm{C} 1 \mathrm{C} 3$ & 12 & \\
\hline & & D3 & $\mathrm{C} 3+$ & $\mathrm{C} 1 \mathrm{C} 2$ & 12 & 36 \\
\hline A D MISTA & & & & & & \\
\hline & Verde & A 1 & D1+ & $\begin{array}{l}\mathrm{D} 2 \mathrm{D} 3 \\
\mathrm{D} 2 \mathrm{D} 4\end{array}$ & $\begin{array}{l}02 \\
02\end{array}$ & \\
\hline & & & $\begin{array}{l}\text { D1t } \\
\text { D1t }\end{array}$ & D 3D 4 & 02 & \\
\hline A 1D 2 & Vermelho & A1 & $\mathrm{D} 2+$ & D1D3 & 02 & \\
\hline & & & D2+ & D1D 4 & 02 & \\
\hline & & & D2t & D 3D 4 & 02 & \\
\hline A 2D 1 & Vermelho & A2 & D1+ & D2D3 & 02 & \\
\hline & & & D1+ & D2D 4 & 02 & \\
\hline & & & D1+ & D 3D 4 & 02 & \\
\hline A 2D2 2 & Verde & A2 & $\mathrm{D} 2+$ & D1D3 & 02 & \\
\hline & & & D2+ & D1D 4 & 02 & \\
\hline & & & D2+ & D 3D 4 & 02 & \\
\hline A 2D 3 & Vermelho & A2 & D3t & D1D2 & 02 & \\
\hline & & & D3+ & D1D 4 & 02 & \\
\hline & & & D3+ & D2D 4 & 02 & \\
\hline A 3D 2 & Vermelho & A3 & $\mathrm{D} 2+$ & D1D3 & 02 & \\
\hline & & & D2+ & D1D 4 & 02 & \\
\hline & & & D2t & D3D 4 & 02 & 36 \\
\hline EQUIV DC & & & & & & \\
\hline $\mathrm{D} 1 \mathrm{C} 1$ & Vermelho & D1 & $\mathrm{C} 1+$ & $\mathrm{C} 2 \mathrm{C} 3$ & 36 & 36 \\
\hline $\mathrm{D} 2 \mathrm{C} 2$ & Vermelho & D2 & $\mathrm{C} 2+$ & $\mathrm{C} 1 \mathrm{C} 3$ & 36 & 36 \\
\hline D3C3 & Vermelho & D3 & C3t & $\mathrm{C} 1 \mathrm{C} 2$ & 36 & 36 \\
\hline
\end{tabular}

SIMET: Simetria

EQUIV: Equivalência das discriminações condicionais, indicando o controle pela consistência modelo-estímulo de comparação correto. Por exemplo, um participante (NET) verbalizou que "associava a figura do centro com a figura que aparecia sempre". Outro participante (PAUL), afirmou que "tocava a figura que não desaparecia da tela".

Três participantes (NET, ALIN e PAUL) demonstraram todas as relações simétricas na ausência do controle contextual e a relação simétrica DA sob controle contextual. Dois deles (NET e ALIN) mostraram essas relações emergentes, na primeira apresentação do bloco de testes, enquanto PAUL mostrou as relações simétricas BA e DA, respectivamente, na ausência e presença do controle contextual, somente na segunda exposição ao bloco de testes.

Nenhum dos participantes, excetuando PAUL, formou as relações de equivalência testadas na ausência do controle contextual (BC, CB e BD). Na relação CB, o participante PAUL mostrou um elevado número de tentativas corretas (ver Tabela 1), apresentando 34 e 33 tentativas, na primeira e segunda apresentações, respectivamente. Em todos os testes de equivalência seguintes (DB, CD e DC), aplicados na ausência do controle contextual, o participante PAUL atingiu o critério consistente com a formação de classes. No entanto, este participante não mostrou a última relação de equivalência DC testada sob controle contextual, mas apresentou o controle contextual da relação de equivalência DB, na repetição deste teste.

Quanto ao controle contextual da discriminação condicional ensinada (AD), todos os participantes atingiram o critério de acertos na primeira ou segunda apresentação do bloco de treino. No entanto, três deles (NET, ALIN e KAR) não demonstraram o controle contextual das relações de equivalência DB e DC, nem na reexposição ao bloco de teste.

O participante KAR não apresentou nenhuma relação emergente (obteve um total máximo de 12 acertos em todos os testes).

\section{Discussão}

Os resultados do presente estudo corroboram os obtidos em estudos anteriores, revelando através de um treino de pareamento consistente modelo-estímulo de comparação correto, o estabelecimento de discriminações condicionais e a emergência de relações simétricas (Baptista \& Assis, 1995; Assis \& cols., 1997; Barros \& cols., 1998 e Damin \& cols., 1998). Os resultados indicaram, ainda, que este procedimento estabeleceu o controle contextual da discriminação condicional $\mathrm{AD}$ ensinada e a relação simétrica DA, na ausência de conseqüências diferenciais imediatas para respostas corretas e incorretas. Estes resultados estendem os relatados por Lazar \& Kotlarchyk, 1986; Wulfert \& Hayes, 1988; Bush, Sidman \& de Rose, 1989; Gatch \& Osborne, 1989; Lynch \& Green, 1991; Assis \& Galvão, 1996, os quais utilizaram consequiências diferenciais explícitas e imediatas para o estabelecimento do controle contextual das discriminações ensinadas e emergentes. 
Tabela 1. Número de tentativas corretas para cada participante, nas relações condicionais de treino misto (TM) e testes de simetria (SIM) e de equivalência (EQ).

\begin{tabular}{|c|c|c|c|c|c|c|c|c|c|c|c|c|c|c|c|c|c|c|c|c|c|c|c|c|}
\hline \multirow[b]{2}{*}{ Participante } & \multirow[b]{2}{*}{$\begin{array}{l}T M \\
A B\end{array}$} & \multirow[b]{2}{*}{$\begin{array}{c}\text { SIM } \\
\text { BA }\end{array}$} & \multicolumn{11}{|c|}{ Contexto } & \multicolumn{8}{|c|}{ Contexto } & \multicolumn{3}{|c|}{ Contexto } \\
\hline & & & $\begin{array}{l}\text { TM } \\
\text { AC }\end{array}$ & $\begin{array}{l}\text { SIM } \\
\text { CA }\end{array}$ & $\begin{array}{l}T M \\
A D\end{array}$ & $\begin{array}{l}\text { SIM } \\
\text { DA }\end{array}$ & $\begin{array}{c}\text { TM* } \\
\text { AD }\end{array}$ & $\begin{array}{c}\text { SIM* } \\
\text { DA }\end{array}$ & $\begin{array}{l}T M \\
A B\end{array}$ & $\begin{array}{l}\text { TM } \\
\text { AC }\end{array}$ & $\begin{array}{l}\text { EQ } \\
\text { BC }\end{array}$ & $\begin{array}{l}\text { EQ } \\
\text { C B }\end{array}$ & $\begin{array}{l}T M \\
A B\end{array}$ & $\begin{array}{l}\text { TM } \\
\text { AD }\end{array}$ & $\begin{array}{l}\text { EQ } \\
\text { BD }\end{array}$ & $\begin{array}{l}\text { EQ } \\
\text { DB }\end{array}$ & $\begin{array}{c}\text { TM* } \\
\text { AD }\end{array}$ & $\begin{array}{c}\text { EQ** } \\
\text { DB }\end{array}$ & $\begin{array}{l}\text { TM } \\
\text { AC }\end{array}$ & $\begin{array}{l}T M \\
A D\end{array}$ & $\begin{array}{l}\text { EQ } \\
C D\end{array}$ & $\begin{array}{l}\text { EQ } \\
\text { DC }\end{array}$ & $\begin{array}{c}\text { TM* } \\
\text { AD }\end{array}$ & $\begin{array}{c}E Q^{* *} \\
\text { DC }\end{array}$ \\
\hline \multirow[t]{2}{*}{ NET } & 35 & 36 & 36 & 36 & 35 & 36 & 35 & 35 & 36 & 36 & 11 & 12 & 36 & 36 & 3 & 12 & 36 & 12 & 36 & 36 & 14 & 12 & 18 & 12 \\
\hline & & & & & & & & & & & 12 & 12 & & & 0 & 12 & & 12 & & & 14 & 12 & 35 & 18 \\
\hline \multirow[t]{2}{*}{ ALIN } & 36 & 35 & 36 & 36 & 21 & 36 & 35 & 35 & 36 & 36 & 24 & 3 & 36 & 36 & 12 & 12 & 36 & 24 & 36 & 36 & 22 & 23 & 23 & 12 \\
\hline & & & & & 36 & & & & & & 24 & 4 & & & 12 & 12 & & 23 & & & 24 & 22 & 36 & 16 \\
\hline \multirow[t]{2}{*}{ KAR } & 26 & 12 & 36 & 12 & 36 & 12 & 14 & 9 & 36 & 36 & 12 & 12 & 36 & 36 & 12 & 12 & 20 & 12 & 36 & 36 & 12 & 12 & 26 & 11 \\
\hline & 36 & 12 & & 12 & & 12 & 35 & 11 & & & 12 & 12 & & & 12 & 12 & 35 & 18 & & & 16 & 22 & 35 & 10 \\
\hline \multirow[t]{2}{*}{ PAUL } & 36 & 29 & 36 & 36 & 35 & 36 & 34 & 18 & 36 & 36 & 10 & 34 & 36 & 35 & 12 & 36 & 35 & 33 & 36 & 35 & 36 & 35 & 35 & 16 \\
\hline & & 36 & & & & & 35 & 36 & & & 12 & 33 & & & 12 & & & 36 & & & & & & 15 \\
\hline
\end{tabular}

$*$ Verde e Vermelho $\quad * *=\mathrm{DB}$ (Verde) DC (Vermelho)

De acordo com Lynch e Green (1991), para ocorrer um verdadeiro controle condicional de segunda ordem, é preciso que as relações condicionais sejam ensinadas sem estímulos contextuais, para depois serem estabelecidas com estímulos contextuais. Os resultados do presente estudo indicaram o estabelecimento de discriminações condicionais e relações simétricas sob controle contextual, através de um treino com pareamento consistente entre o modelo e o estímulo de comparação correto, na ausência de conseqüências diferenciais imediatas, utilizando uma seqüência de treino semelhante a descrita por Lynch e Green (1991).

As relações de equivalência previstas não ocorreram prontamente e nem após a reexposição aos testes, para três participantes. No entanto, os resultados de um participante (PAUL) sugerem a emergência atrasada (delayed emergence, segundo Sidman, 1994) das relações de equivalência testadas (DB) e que se manteve em duas outras relações de equivalência testadas posteriormente (CD e DC). Para este participante, houve controle contextual somente na equivalência (DB), após a repetição do teste. A ausência ou o fraco controle contextual pode ter decorrido das dificuldades no seu estabelecimento devido ao tipo de tentativas de treino. Por exemplo, nas tentativas de treino para estabelecer o controle contextual das discriminações A2D1 e A2D3 (AD mista) na presença do vermelho (ver Quadro 3 e 4). Nestes dois tipos de tentativas, o participante era exposto à mesma configuração dos estímulos - na presença da cor vermelha, modelo A2 e estímulos de comparação D1, D2 e D3, ele deveria escolher D1 (tentativa A2D1) e D3 (tentativa A2D3). A única diferença entre estes dois tipos de tentativas era a apresentação consistente modelo-estímulo de comparação correto que poderia se tornar evidente somente após a apresentação de várias tentativas, quando um dos estímulos de comparação incorreto mudava a cada 2 tentativas. Além disto, a exposição anterior à tentativas do tipo A2D1 sob controle contextual da cor vermelha pode ter interferido no estabelecimento da relação A2D3 sob controle da mesma cor.

Outra dificuldade que pode ter dificultado a emergência das relações de equivalência e o controle contextual dessas relações, refere-se ao procedimento de treino por consistência. O estímulo de comparação correto era apresentado consistentemente com o modelo durante o treino e um dos estímulos de comparação mudava a cada duas ten- tativas. Esta permanência de um dos estímulos de comparação incorreto em duas tentativas consecutivas pode ter dificultado a formação destas relações emergentes e o controle contextual pelas cores. Além disto, a diferença no número de vezes em que cada estímulo de comparação incorreto era apresentado simultaneamente com o modelo pode ter interferido no controle exercido pelo estímulo de comparação correto, que era apresentado consistentemente com o modelo, e pelos estímulos de comparação incorretos apresentados de forma inconsistente.

A formação de relações emergentes (simétricas), após treino consistente envolvendo estímulos contextuais, verificada no presente experimento, evidencia, mais uma vez, um forte controle experimental derivado do arranjo de treino utilizado, mesmo quando não tinham sido programadas consequiências diferenciais para as respostas "corretas" ou "incorretas" em cada tentativa.

O presente estudo tem pelo menos duas importantes implicações. Primeiramente, a evidência de controle contextual das relações ensinadas e simétricas constitui uma ampliação dos estudos que mostraram a eficácia do procedimento de pareamento consistente entre modelo e estímulo de comparação correto no estabelecimento de discriminações condicionais e emergência de relações simétricas entre estímulos (Harrison \& Green, 1990; Baptista \& Assis, 1995; Álvares, 1996; Assis \& cols., 1997; Barros \& cols., 1998; Damin \& cols., 1998). Os resultados obtidos estendem os de estudos anteriores que utilizaram conseqüências diferenciais imediatas para estabelecer o controle contextual das discriminações condicionais ensinadas e das relações emergentes (Lazar \& Kotlarchyk, 1986; Wulfert \& Hayes, 1988; Bush, Sidman \& de Rose, 1989; Gatch \& Osborne, 1989; Lynch \& Green, 1991; Assis \& Galvão, 1996). A segunda implicação, é que este estudo pode ser visto como uma alternativa de investigação do controle de estímulos e das fontes de reforço mantenedoras do desempenho durante o treino e durante os testes, na ausência de conseqüências diferenciais imediatas.

Conforme apontado por Sidman (1992) e Harrison \& Green (1990), dependendo da estrutura e do balanceamento das tentativas de teste, o participante pode ser exposto ao treino direto e demonstrar relações condicionais durante $o$ teste. Isso foi evidenciado no presente estudo. Os resultados 
do presente estudo indicam ainda que as relações estabelecidas no treino e as emergentes podem ficar sob controle de estímulos contextuais presentes na situação de teste.

A forma como os estímulos condicional e discriminativo são dispostos, isto é, caracterizando o arranjo de treino consistente em cada tentativa, pode produzir um desempenho que se mantém nos testes de relações emergentes, não somente quando esse arranjo é simples mas também quando reflete a complexidade que caracteriza o controle contextual, verificado no presente estudo. Num caso ou noutro, a relação entre estímulos (discriminativo, condicional e contextual) parece ser a relação pertinente a ser considerada na análise do desempenho. Ademais, em ambos os casos, apenas após reapresentações de blocos de tentativas é possível que o participante constate seus "erros" ou "acertos", o que não se verifica em cada tentativa, pela ausência de consequiências diferenciais em cada uma delas. A reapresentação desses blocos, prevista neste e nos demais estudos similares, ocorre com sua maior frequiência pelo menos no treino inicial e parece fornecer pistas ou dicas indicadoras de "erros" ou "acertos". As pistas ou dicas, que são tardias, dada a sua provável existência após reapresentações de tentativas, podem funcionar como estímulos discriminativos e parecem controlar o responder como as regras o fazem.

É importante ressaltar que conseqüências diferenciais tardias foram programadas para respostas corretas e incorretas, ou seja, depois de um determinado bloco de treino, a mudança no tipo de tentativa poderia indicar ao participante que seu desempenho era correto ou incorreto. Após um bloco de trinta e seis tentativas consecutivas corretas, mudava o tipo de tentativa e estímulos. O desempenho correto pode ter relação com alguma auto-regra formulada (ver os relatos de NET e PAUL na entrevista), que levasse em conta a consistência na relação entre estímulos, à semelhança de como procederam os participantes do estudo de Baptista \& Assis (1995), os quais expressaram, em entrevista pósexperimental, hipóteses levantadas durante as sessões e confirmadas por eles, acerca das relações entre modelos e estímulos de comparação que eram consistentes e das relações que eram inconsistentes. No presente estudo, conduzido na ausência de conseqüências diferenciais imediatas, o controle do desempenho parece restringir-se à forma como as relações entre os estímulos contextual, condicional e discriminativo foram organizados. $\mathrm{O}$ desempenho parece ter-se estabelecido e se mantido mesmo na ausência de conseqüência diferencial imediata para a sua ocorrência e sensível ao controle dos estímulos que o antecediam.

\section{Referências}

Álvares, S.M.M. (1996). Discriminações condicionais sem conseqüiencias diferenciais em crianças: treino por fading e teste precoce de simetria. Dissertação de Mestrado, Universidade Federal do Pará.

Assis, G.J.A. \& Galvão, O.F. (1996). Relações condicionais entre palavras conhecidas. Acta Comportamentalia, 4 (1), 522.
Assis, G.J.A. Baptista, M.Q.G. Damin, E.T.B. \& Álvares, S.M.M. (1997). Consistency training and equivalence relations without differential consequences. Experimental Analysis of $\mathrm{Hu}$ man Behavior Bulletin. 15 (1), 6-7.

Baptista, M.Q.G. \& Assis, G.J.A. (1995). Treino por consistência de estímulos sem conseqüências diferenciais. Psicologia: Teoria e Pesquisa, 11(3), 173-179.

Barros, C.W., Baptista, M.Q.G. \& Assis, G.J.A. (1998). Efeitos da história de treino na formação de classes de estímulos equivalentes. Acta Comportamentalia, 6(2), 111-128.

Bush, K.M., Sidman, M., \& de Rose, T. (1989). Contextual control of emergent equivalence. Journal of the Experimental Analysis of Behavior, 51 (1), 29-45.

Catania, A.C. (1968). Contemporary Research in Operant Behavior. Glenview, Illinois: Scott, Foresman and Company.

Catania, A.C. (1984). Learning (2nd), Englewood Cliffs, N.J: Prentice-Hall.

Damin, E.T.B., Assis, G.J.A. \& Baptista, M.Q.G. (1998). Efeitos da distribuição de treino e testes na formação de classes de estímulos equivalentes. Psicologia, Teoria e Pesquisa., 14(1), 41-49.

Gatch, M.B. \& Osborne, J.G. (1989) Transfer of contextual stimulus function via equivalence class development. Journal of the Experimental Analysis of Behavior, 51(3), 369-378.

Harrison, R.J. \& Green, G. (1990). Development of conditional and equivalence relations without differential consequences. Journal of the Experimental Analysis of Behavior, 54(3), 225237.

Lazar, M.R. \& Kotlarchyk, B.J. (1986). Second-order control of sequence-class equivalence in children. Behavioural Processes, 13, 205-215.

Lynch, D.C. \& Green, G. (1991). Development and crossmodal transfer of contextual control of emergent stimulus relations. Journal of the Experimental Analysis of Behavior, 56(1), 139154.

Reynolds, G.S. (1975). A primer of operant conditioning. Glenview, Illinois: Scott Foresman and Company.

Rilling, M. (1977). Stimulus control and inhibitory processes. Em: W.K. Honing \& J.E.R Staddon (Orgs.), Handbook of operant behavior (pp.432-480). Englewood Cliffs, NJ: PrenticeHall.

Saunders, R.S., Saunders, K.J., Kirby, K.C., \& Spradlin, J. E. (1988). The merger and development of equivalence classes by unreinforced selection of comparison stimuli. Journal of the Experimental Analysis of Behavior, 50(2), 145-161.

Schlinger Jr., H.D., Blakely, E., Fillhard, J. \& Poling, A. (1991). Defining Terms in Behavior Analysis: Reinforcer and Discriminative Stimulus. The Analysis of Verbal Behavior, 9, 153161.

Schoenfeld, W.N. (1995). "Reinforcement" in behavior analysis. The Behavior Analyst, 18 (1), 173-185.

Skinner, B.F. (1953). Science and Human Behavior, New York: The Macmillan Company.

Sidman, M. (1986). Funtional analysis of emergent verbal classes. Em: T. Thompson and M.D.Zeiler (Orgs.), Analysis and integration of behavioral units (pp. 213-245). Lawrence Erlbaum, Hillsdale, N.Y. 
Sidman, M. (1992). Equivalence relations: some basic considerations. Em: S.C.Hayes \& L.J. Hayes (Orgs.), Understanding verbal relations: The Second and Third International Institute on Verbal Relations (pp. 15-27). Reno, Nevada: Context Press.

Sidman, M. (1994). Equivalence Relations and Behavior: a research story. Boston, MA: Authors Cooperative Publisherrs.
Sulzer-Azaroff, B \& Mayer, G.R. (1977). Applying behavioranalysis procedures with children and youth. New York: Holt, Rinehart, and Winston.

Wulfert, E. \& Hayes, S.C. (1988). Transfer of a conditional ordering response through conditional equivalence classes. Journal of the Experimental Analysis of Behavior, 50 (20), 125144. 